\title{
Healthcare Resource Utilization for Children Requiring Prolonged Mechanical Ventilation via Tracheostomy
}

\author{
Colin Rogerson, MD, Andrew Beardsley, MD, MS, \\ Mara Nitu, MD, MBA, Ioana Cristea, MD, MS
}

\begin{abstract}
Affiliations: Riley Hospital for Children, Indianapolis, IN
Address Correspondence to: Colin Rogerson, Division of Pediatric Critical Care, Riley Hospital for Children, 705 Riley Hospital Drive, Indianapolis, Indiana, 46234, (crogerso@iupui.edu),317-944-5000.
\end{abstract}

\section{Author List:}

Dr. Colin M. Rogerson, MD. Riley Hospital for Children, Indiana University. Pediatric Critical Care Division. Indianapolis, IN. USA.

Helped with the project conception and design, literature search, submitted the IRB, designed the data collection tool, collected the patient data, conducted the statistical analysis of the data, drafted the initial manuscript, and revised the manuscript.

Dr. Andrew L. Beardsley, MD, MS. Riley Hospital for Children, Indiana University. Pediatric Critical Care Division. Indianapolis, IN. USA.

Helped with the project conception and design, reviewed and revised the IRB, reviewed the statistical analysis, and helped format the results.

Dr. Mara E. Nitu, MD, MBA. Riley Hospital for Children, Indiana University. Pediatric Critical Care Division. Indianapolis, IN. USA.

Helped with the project conception and design, and reviewed and revised the manuscript.

Dr. A. Ioana Cristea, MD, MS. Riley Hospital for Children, Indiana University. Pediatric Pulmonology Division. Indianapolis, IN. USA.

Helped with the project conception and design, literature search, provided the patient lists for data collection, and reviewed and revised the manuscript.

\section{Previous Presentations:}

March 2019 PALISI (Pediatric Acute Lung Injury and Sepsis Investigators) chronic ventilation subgroup meeting in Snowbird, UT

April 2019 PAS (Pediatric Academic Society) large forum poster session in Baltimore, MD

The authors have no financial conflicts of interest to disclose. This study had no national funding sources. All authors approved the final manuscript as submitted and agree to be accountable for all aspects of the work.

This is the author's manuscript of the article published in final edited form as:

Rogerson, C. M., Beardsley, A. L., Nitu, M. E., \& Cristea, A. I. (2020). Health care resource utilization for children requiring prolonged mechanical ventilation via tracheostomy. Respiratory Care, 65(8), 1147-1153. https://doi.org/10.4187/respcare.07342 


\begin{abstract}
$\underline{\text { Abstract }}$
Background: More children are discharged from ICUs on prolonged mechanical ventilation via tracheostomy than ever before. These patients have long hospitalizations with high resource expenditure. Our objective was to describe the characteristics of these resource-intensive patients, and to evaluate their costs of care. We hypothesized that subjects requiring prolonged mechanical ventilation for neurologic diagnoses would have higher costs, longer hospital length of stay (LOS), and worse outcomes than those with primarily respiratory diagnoses.
\end{abstract}

Methods: We identified 50 pediatric subjects with a new tracheostomy placement between January 2015 and December 2017 at our institution, and were enrolled in a home mechanical ventilation program. Collected data included demographics, indication for tracheostomy, LOS, hospital costs, readmissions, and outcomes. We also compared subjects who required prolonged mechanical ventilation for respiratory diagnoses vs. neurologic diagnoses.

Results: Of our 50 subjects, 41 were less than 12 months old at the time of tracheostomy. Thirtyfour had a respiratory diagnosis requiring prolonged mechanical ventilation, 14 had a neurologic diagnosis, and 2 had a cardiac diagnosis. The total initial hospitalization cost was $\$ 31,133,582$, which averages to $\$ 622,671$ per subject. The average initial hospitalization LOS was 155 days. Respiratory subjects had longer LOS and higher average costs than neurologic patients. The average readmission rate was 2.16 per subject in the first year after discharge, and the average readmission cost per subject was $\$ 170,964$. Eight subjects died in the first year after discharge, and 4 suffered a serious morbidity.

Conclusions: This descriptive study evaluated the social and medical characteristics of subjects being discharged from the PICU with prolonged mechanical ventilation via tracheostomy, as well as quantified the financial impact of their care. Those requiring prolonged mechanical 
ventilation for neurologic diagnoses had shorter hospital LOS and lower hospital costs than those with respiratory diagnoses. No definitive differences in outcomes were found.

\section{Introduction:}

An increasing number of children with complex medical conditions survive their critical illness with the support of prolonged mechanical ventilation via tracheostomy tubes. ${ }^{1-5}$ Medical advancements over the past few decades have made this a safe and effective approach to managing these patients either at home or in long-term health care facilities. ${ }^{4,9}$ Previous studies have shown that patients requiring prolonged mechanical ventilation have a high rate of complications and hospital readmissions, and the financial costs associated with caring for them can be a large burden to the health care system. ${ }^{3,10-15}$

There is insufficient data describing the healthcare utilization of pediatric patients requiring prolonged mechanical ventilation. The typical initial hospitalization includes a long initial ICU admission followed by considerable additional hospital time dedicated to arduous

training of the caregivers to safely care for these patients at home. ${ }^{14,16}$ While hospital length of stay (LOS) has been described previously, ${ }^{13,14}$ few studies have quantified the total healthcare utilization. For many patients, despite prolonged hospitalizations and the utilization of large amounts of health care resources, readmissions are a frequent occurrence and long-term outcomes are often poor. ${ }^{10,13,14,17}$ While it has been proposed that there is a difference in both costs and outcomes associated with different underlying conditions leading to the need for prolonged mechanical ventilation, a comparison between these patients has been described in only a few studies. ${ }^{18-20}$ 
Our objective was to describe the social and medical characteristics of these resourceintensive patients, and to evaluate the cost of caring for them both for the initial hospital admission, and for readmissions in the first year after discharge. We hypothesized that subjects requiring prolonged mechanical ventilation for neurologic diagnoses would have higher costs, longer hospital LOS, and worse outcomes than those with primarily respiratory diagnoses.

\section{Methods:}

This is a retrospective cohort study of subjects under 18 years of age who underwent new tracheostomy placement, and were discharged on prolonged mechanical ventilation between January 1, 2015 and December 31, 2017 at Riley Hospital for Children in Indianapolis, Indiana. Subjects were excluded if they received a tracheostomy but were not discharged on prolonged mechanical ventilation, if they were re-cannulated after a previous tracheostomy, or if they had a tracheostomy performed at another institution and were then transferred to our institution for initiation of prolonged mechanical ventilation. Also, subjects who died during their initial hospitalization were excluded from the study. This study plan was submitted to the Indiana University IRB and was granted exemption.

Our institution's current process for patients admitted to the NICU that are identified as requiring prolonged mechanical ventilation involves transferring to the PICU prior to tracheostomy placement, and afterwards transitioning from a hospital ventilator to a home mechanical ventilator. Once the subject is stable on a home mechanical ventilator, medical care is transitioned to a chronic vent team which consists of pulmonary nurse practitioners, social worker, respiratory therapist and pediatric pulmonary attending physicians. While our program 
is located within the PICU geographical space, it is cohosted in a special 8 bed space which functions as a Chronic Vent Unit where patients are transitioned to home ventilation and caregivers are receiving training on their child's needs. The staffing is provided by a separate dedicated RT as well as dedicated RNs with expertise in training these subjects for home discharge. The regular PICU RNs and RTs are not included in the staffing pool for this unit. Parents and/or caregivers are trained in routine and emergent tracheostomy care as well as home ventilator management. Our institution follows the ATS guidelines for discharge to home on prolonged mechanical ventilation, which requires a minimum of two full-time caregivers to undergo this training and be available at all times to care for these subjects. ${ }^{21}$ Subjects that are admitted to the PICU and are then identified as requiring prolonged mechanical ventilation via tracheostomy tube follow the same process.

All subject data was collected from electronic medical records (Cerner Corporation, Kansas City, MO). Subjects' demographic data included age, gender, ethnicity, primary language of subject and/or caregivers, marital status of primary caregiver, age of mother at time of subject's birth, mother's employment status, and identity of the second trained caregiver. Some subjects changed their second caregiver, and if this was done more than once they were classified as having difficulty identifying a second caregiver. Hospital course dates and other dates were obtained including date of birth, admission date, date of decision to proceed with tracheostomy, date of tracheostomy, date of first tracheostomy tube change, date of PICU transfer (if applicable), date of transfer to the chronic vent team, and date of discharge. Medical data for each subject was collected and included the subjects' gestational age, primary diagnosis leading to the need for tracheostomy and prolonged mechanical ventilation which was categorized into broad labels including "Respiratory", "Neurologic", and "Cardiac", and the 
surgical service performing the tracheostomy. Discharge follow-up data included subjects' discharge location (home vs. chronic care facility), Department of Child Services (DCS) involvement, which is Indiana's State Government agency responsible for addressing child abuse or neglect, and home skilled nursing availability. Outcome data was obtained for the first year post hospital discharge, which included subjects' ED visits, number of readmissions, hospitaldays per readmission, mortality, and serious morbidity (which included survived cardiac arrest, stroke, anoxic brain injury, or unplanned hospital admission requiring surgical intervention). Financial data was also obtained from Medicaid for each subject, as all of our cohort subjects were supported by Indiana Medicaid. This data included all Medicaid charges for each subject's initial hospitalization and for subsequent readmissions to Indiana University facilities. Costs to the hospital were also obtained, and are defined as a hospital specified factor of each Medicaid charge. Itemized charges related to nursing, radiology, respiratory, pharmacy, lab, physical and occupational therapy, surgery, pulmonary, and emergency charges were collected. All financial data represents direct costs which include procedures, medications, imaging, etc. that are directly provided to the subject, and does not include indirect costs such as hospital staff training, insurance, quality assurance fees, etc. Collected data was stored in REDCap for HIPAA compliance. All descriptive statistics were obtained using RStudio version 3.4.3.

We also performed a sub-analysis separating the subjects who required prolonged mechanical ventilation via tracheostomy for primarily respiratory diagnoses from those for primarily neurologic diagnoses. We obtained the same descriptive statistics for these groups as was done for the full cohort of 50 subjects. This sub-analysis excluded the subjects with primarily cardiac diagnoses. Sub-analysis comparisons were performed using Mann-Whitney U tests in RStudio version 3.4.3. 


\section{Results:}

Subjects were identified using lists acquired from the Home Vent Program at Riley Hospital. Between January 1, 2015 and December 31, 2017 a total of 87 subjects were identified. Fourteen of these subjects were excluded because we were unable to obtain a full financial analysis from Medicaid at the time the study was performed, as the one-year follow-up data was not available. Eight subjects were excluded due to the subjects' tracheostomy being performed at another hospital prior to transfer to our institution. Seven subjects had the tracheostomy performed prior to 2015 and were discharged in 2015. Four subjects had a tracheostomy placed prior to admission, and were only admitted to initiate prolonged mechanical ventilation. Two subjects were enrolled in the chronic ventilation program, but were discharged on trach collar only. One subject was admitted only for re-cannulation after previously being decannulated. One subject died prior to discharge. This left a final cohort of 50 subjects who were included in the study analysis.

Table 1 outlines the general characteristics of our study population. The majority of our subjects $(82 \%)$ were less than one year of age at the time of their tracheostomy placement, with a median age of four and a half months. Table 2 displays the characteristics of the sub-groups, separated by the primary diagnosis requiring prolonged mechanical ventilation via tracheostomy tube. The majority of our subjects $(68 \%)$ required prolonged mechanical ventilation via tracheostomy tube for a primarily respiratory diagnosis. Eighteen of these subjects had bronchopulmonary dysplasia (BPD), five had chronic lung disease, four had congenital diaphragmatic hernias, three had acute respiratory distress syndrome, three had pulmonary hypoplasia, and one had severe obstructive sleep apnea as the subject's primary respiratory diagnosis. Of those subjects with primarily neurologic diagnoses, four had a neuromuscular 
disorder, four had a genetic or metabolic syndrome, four had severe cerebral palsy, and two had hypoxic ischemic encephalopathy. On average, the respiratory group was younger at the time of tracheostomy placement than the neurologic group (Median 4.5 months uncorrected for gestational age vs. 24 months). The two cardiac subjects were such a small proportion of our population that although they are included in the total analysis, they were not analyzed as a separate subgroup.

Table 3 shows the average time in days between major events in our population as they progressed through initial hospitalization. The first time point is the time between hospital admission and the tracheostomy placement. Second is the time between the medical team and family coming to a decision to proceed with a tracheostomy placement and the procedure occurring. Third is the time between the tracheostomy placement and the first tracheostomy tube change. The final time is between the tracheostomy placement and discharge from the hospital. The comparison p-value is between the two subgroups, and showed the respiratory group with significantly longer average time between hospital admission and tracheostomy placement, as well as between tracheostomy placement and hospital discharge. Other hospital characteristics include the majority of subjects being admitted initially to the NICU rather than the PICU (72\% vs. $26 \%$ ), and only one subject admitted to the floor. Pediatric ENT performed the majority of the tracheostomies compared to pediatric general surgery ( $78 \%$ vs. $22 \%$ ).

Table 4 illustrates both the initial hospitalization costs and length of stay data for our subject population. The total data for the whole population is given, as well as the subgroup comparison. The neurologic subjects had significantly lower average hospital costs and length of stay. 
When evaluating the itemized charges for these subjects, we found that the respiratory subjects had significantly higher charges for nursing ( $\mathrm{p}$-value $<.01$ ), respiratory ( $\mathrm{p}$-value $<.01$ ), pharmacy (p-value .02), physical therapy and occupational therapy (p-value $<.01$ ), and laboratory care ( $\mathrm{p}$-value $<.01)$ than did the neurologic subjects. However, when adjusting for the subjects' overall LOS, and calculating these charges per day, these differences were not seen. All itemized charges, when measured per patient day, were found to have no significant difference between the two groups. There was no difference in the charges for radiology, surgery, pulmonology, or emergency care between the two groups.

Our study population had variability in their discharge destination as well as availability of home nursing. Forty-five (90\%) of our subjects were discharged to home and the remaining five $(10 \%)$ were discharged to a long-term care facility. Thirty-six $(72 \%)$ of our subjects had home nursing approved prior to discharge. Eight subjects (16\%) had DCS involvement at some point during their initial hospitalization.

Table 5 displays the readmission rate, costs, and hospital-free days of our subject population. Although it appears that the neurologic subjects have higher readmission costs and fewer hospital-free days, these comparisons were not significantly different given our current data (p-value .07). It is possible, however, that a larger sample size may provide the statistical power to detect a difference.

Eight subjects died within the first year of discharge ( $16 \%$ mortality rate). The causes of death included three compassionate withdrawals of mechanical support (One in the setting of a newly diagnosed critical illness, and two as the families did not appreciate any significant recovery and chose to allow natural death), two due to tracheostomy tube dislodgement leading to respiratory and subsequently cardiac arrest, two due to cardiac arrests at home of uncertain 
etiologies and one due to stroke that left the subject brain dead. Mortality rates were $12 \%$ in the respiratory group (4/34) compared to $29 \%$ in the neurologic group (4/14). These rates were not significantly different on hypothesis testing with a p-value of 0.2 . A total of 12 subjects had a serious morbidity which was defined as either a successfully resuscitated cardiac arrest, trach dislodgement leading to respiratory arrest, a stroke or hypoxic-ischemic encephalopathy, an unplanned ICU admission requiring a surgical intervention, or death. The serious morbidity rates were also different with six subjects in each group having a serious morbidity. This gives rates of $18 \%$ for the respiratory group and $43 \%$ for the neurologic group, which was also shown to be non-significant on hypothesis testing with a p-value of .14. It is likely, however, that both the mortality and serious morbidity rates are different between the respiratory and neurologic groups, but a larger sample size is needed to adequately detect them.

\section{Discussion:}

This study not only offers a rare epidemiological outline for a unique and growing cohort of pediatric patients requiring prolonged mechanical ventilation, but also provides quantifiable data regarding the monumental resource burden this emerging patient population can be to the healthcare system. In regards to the epidemiology of our study, our findings are similar to previous studies showing that the majority of these patients are less than a year old, and require prolonged mechanical ventilation for the management of primarily respiratory diseases. ${ }^{20,22}$ Our financial data showed that the average cost to the hospital for caring for a single child requiring a tracheostomy and prolonged mechanical ventilation was over $\$ 600,000$ for the initial admission. Previous studies, and most intensive-care providers' anecdotal experience, take note quickly of the lengthy admissions and slow progress of this patient population towards discharge. While 
the dramatic hospital length of stays have been touted in the literature, seeing the staggering monetary amount of inpatient hospital costs may motivate providers and hospital administrators to find new ways to help these patients navigate their hospital stays more efficiently. It has been shown that several inpatient and outpatient interventions have been successful in providing more efficient and cost-effective care for this patient population. ${ }^{23,24}$. The results of our study may help hospital systems prioritize these types of interventions in the future, to provide not only more efficient care, but also more effective care for these patients.

The one year follow-up data we are describing is consistent with previous literature reports. These patients had frequent and costly readmissions with more than three-quarters of our subjects being readmitted during the first year. The one-year mortality rate of these subjects was also relatively high compared to the general population at $16 \%$, and while lower than the rates reported in a few other studies of this population, this is only a one-year mortality rate while other studies provide 5 or more year mortality rates. ${ }^{12,25,26}$ This continues to emphasize that despite long hospitalizations requiring rigorous caregiver training and copious resource utilization, these patients continue to struggle with their underlying diseases as well as the risks inherent with managing a home ventilator.

By analyzing the data between respiratory and neurologic subgroups, we were able to demonstrate several differences between the two. The patients with neurologic conditions were typically older than those with respiratory conditions. This is likely due to the higher number of premature infants with bronchopulmonary dysplasia who more frequently require tracheostomies and prolonged mechanical ventilation. We also found, contrary to our initial hypothesis, that on average, children with primarily respiratory illnesses have longer length of stays and higher hospital costs than those with primarily neurologic illnesses. There are likely many underlying 
reasons for this difference. Again, a large proportion of our subjects requiring prolonged mechanical ventilation are premature infants with BPD who, secondary to their prematurity, require prolonged hospitalization to establish feeding plans, stable growth, and sustained adequate lung function before any attempt at discharge can be made. It can also be difficult for providers to determine which patients with BPD will require a tracheostomy and prolonged mechanical ventilation, as many will have improved lung function over time and can be discharged without the need for prolonged mechanical ventilation. Several of our subjects with neurologic conditions were admitted as teenagers and, due to their declining overall health, required a tracheostomy and prolonged mechanical ventilation. It is often a more straightforward decision for providers to determine if this population will require prolonged mechanical ventilation or not, and so these patients are frequently admitted to the PICU and discharged in far less time than a premature infant with BPD.

There are significant ethical considerations when determining which patients are appropriate for prolonged mechanical ventilation at home, particularly patients with severe neurologic injury. In our organization, all patients are offered a palliative care consult before proceeding with this decision. Furthermore, these patients are discussed in a weekly multidisciplinary team meeting, called PEACE (Pediatric Ethics and Communication Excellence). Palliative care physicians, nurses, respiratory therapists, social workers, medical ethicists, and intensivists attend this meeting. Any concerns the group identifies are discussed by the palliative care and ICU team with the family. ${ }^{27}$

When evaluating the itemized charges for our subjects, we found no significant differences between the two groups when adjusted per subject day. This finding illustrates that 
the subjects' inherent medical conditions are not necessarily the key drivers in determining the overall hospital costs, but is more likely the subjects' length of stay.

Our study has several limitations. It is a retrospective study, which leaves it more prone to bias. Also, our cohort was only fifty subjects which limits the statistical power to detect smaller differences between our two sub-groups. It is of note that several of the subject variables described in this study (Timing of tracheostomy placement, transition of care from NICU to PICU, discharge education, etc.) are all heavily influenced by our institution's specific practice, culture, and unit specific logistics. As a single-center located in the central United States, our findings do lack the capacity for wide generalization, particularly to other healthcare systems outside of the United States. While our study's generalizability is limited, it does provide an example which can be compared to other institutions' experiences and used to add to the overall knowledge of caring for this patient population. We were unable to gather data on length of stay or hospital costs for any subjects who were admitted to a hospital outside of the Indiana University Health system, such as subjects living a large geographic distance from our center. We were also unable to accurately track ED visits for these subjects who presented to outside emergency departments. However, the majority of our subjects live close to our center and were admitted here and nowhere else. A limitation of our cost data is that it does not include physician charges. While we were able to identify which patients had skilled nursing and which did not at discharge, our available data was unable to accurately track the number of hours of skilled nursing available to these families. We also were unable to obtain specific financial data from private insurers for those patients who had private insurance. However, less than $20 \%$ of our subjects had any amount of private insurance, and all were eventually enrolled in Indiana 
Medicaid. A final limitation is that with our data being only from a single center, Medicaid data may be highly variable at other institutions around the country.

\section{Conclusions:}

While the demographics and the medical characteristics of this subject cohort are similar to previous literature reports, we offer the first glimpse into the complicated landscape of the healthcare costs associated with a pediatric cohort of prolonged mechanical ventilation subjects. We conclude that those requiring prolonged mechanical ventilation for primarily neurologic diagnoses had shorter hospital LOS and lower hospital costs than those with primarily respiratory diagnoses. No definitive differences in outcomes were found. This study opens a great opportunity for consideration of care effectiveness projects aimed to reduce these patients' hospital length of stay. 
1. Gowans M, Keenan HT, Bratton SL. The population prevalence of children receiving invasive home ventilation in Utah. Pediatric pulmonology 2007;42(3):231-236.

2. Graham RJ, Fleegler EW, Robinson WM. Chronic ventilator need in the community: a 2005 pediatric census of Massachusetts. Pediatrics 2007;119(6):e1280-1287.

3. Benneyworth BD, Gebremariam A, Clark SJ, Shanley TP, Davis MM. Inpatient health care utilization for children dependent on long-term mechanical ventilation. Pediatrics 2011;127(6):e1533-e1541.

4. Chau SK, Yung AW, Lee SL. Long-Term Management for Ventilator-Assisted Children in Hong Kong: 2 Decades' Experience. Respiratory care 2017;62(1):54-64.

5. Amin RS, Fitton CM. Tracheostomy and home ventilation in children. Seminars in Neonatology;8(2):127-135.

6. Watters K, O'Neill M, Zhu H, Graham RJ, Hall M, Berry J. Two-year mortality, complications, and healthcare use in children with medicaid following tracheostomy. Laryngoscope;126(11):26112617.

7. Reiter K, Pernath N, Pagel P, Hiedi S, Hoffmann F, Schoen C, et al. Risk factors for morbidity and mortality in pediatric home mechanical ventilation. Clinical Pediatrics;50(3):237-243.

8. Fields Al. Pediatric tracheostomy: the great liberator or the last battlefield? Pediatric critical care medicine : a journal of the Society of Critical Care Medicine and the World Federation of Pediatric Intensive and Critical Care Societies 2008;9(1):126-127.

9. Messineo A, Giusti F, Narne S, Mognato G, Antoniello L, Guglielmi M. The safety of home tracheostomy care for children. Journal of Pediatric Surgery;30(8):1246-1248.

10. Meier JD, Valentine KJ, Hagedorn C, Hartling C, Gershan W, Muntz HR, et al. Emergency department use among children with tracheostomies: Avoidable visits. Journal of pediatric rehabilitation medicine 2015;8(2):105-111. 
11. Wilcox $\mathrm{L}$, Weber BC, Cunningham TD, Baldassari CM. Tracheostomy Complications in Institutionalized Children with Long-term Tracheostomy and Ventilator Dependence.

Otolaryngology--head and neck surgery : official journal of American Academy of

Otolaryngology-Head and Neck Surgery 2016;154(4):725-730.

12. Hsia SH, Lin JJ, Huang IA, Wu CT. Outcome of long-term mechanical ventilation support in children. Pediatrics \& Neonatology;53(5):304-308.

13. Kun SS, Edwards JD, Ward SL, Keens TG. Hospital readmissions for newly discharged pediatric home mechanical ventilation patients. Pediatric pulmonology 2012;47(4):409-414.

14. Graf JM, Montagnino BA, Hueckel R, McPherson ML. Pediatric tracheostomies: a recent experience from one academic center. Pediatric critical care medicine : a journal of the Society of Critical Care Medicine and the World Federation of Pediatric Intensive and Critical Care Societies 2008;9(1):96-100.

15. Berry JG, Graham DA, Graham RJ, Zhou J, Putney HL, O'Brien JE, et al. Predictors of clinical outcomes and hospital resource use of children after tracheotomy. Pediatrics 2009;124(2):563572.

16. Sobotka SA, Hird-McCorry LP, Goodman DM. Identification of Fail Points for Discharging Pediatric Patients With New Tracheostomy and Ventilator. Hospital pediatrics;6(9):552-557.

17. Cristea Al, Ackerman VL, Davis SD, Carroll AE, Downs SM, Yu Z, et al. Median Household Income: Association with Mortality in Children on Chronic Ventilation at Home Secondary to Bronchopulmonary Dysplasia. Pediatric allergy, immunology, and pulmonology 2015;28(1):4146.

18. McPherson ML, Shekerdemian L, Goldsworthy M, Minard CG, Nelson CS, Stein F, et al. A decade of pediatric tracheostomies: Indications, outcomes, and long-term prognosis. Pediatric pulmonology 2017;52(7):946-953. 
19. Dursun O, Ozel D. Early and long-term outcome after tracheostomy in children. Pediatrics international : official journal of the Japan Pediatric Society 2011;53(2):202-206.

20. Spentzas T, Auth M, Hess P, Minarik M, Storgion S, Stidham G. Natural course following pediatric tracheostomy. Journal of intensive care medicine 2010;25(1):39-45.

21. Sterni LM, Collaco JM, Baker CD, Carroll JL, Sharma GD, Brozek JL, et al. An Official American Thoracic Society Clinical Practice Guideline: Pediatric Chronic Home Invasive Ventilation. American journal of respiratory and critical care medicine 2016;193(8):e16-35.

22. Appierto L, Cori M, Bianchi R, Onofri A, Catena S, Ferrari M, et al. Home care for chronic respiratory failure in children: 15 years experience. Paediatric Anaesthesia;12(4):345-350.

23. Graham RJ, McManus ML, Rodday AM, Weidner RA, Parsons SK. Pediatric Specialty Care Model for Management of Chronic Respiratory Failure: Cost and Savings Implications and Misalignment With Payment Models. Pediatric Critical Care Medicine 9000;Publish Ahead of Print.

24. Baker CD, Martin S, Thrasher J, Moore HM, Baker J, Abman SH, et al. A Standardized Discharge Process Decreases Length of Stay for Ventilator-Dependent Children. Pediatrics 2016;137(4).

25. Edwards JD, Kun SS, Keens TG. Outcomes and causes of death in children on home mechanical ventilation via tracheostomy: an institutional and literature review. The Journal of pediatrics 2010;157(6):955-959 e952.

26. Cristea Al, Carroll AE, Davis SD, Swigonski NL, Ackerman VL. Outcomes of children with severe bronchopulmonary dysplasia who were ventilator dependent at home. Pediatrics 2013;132(3):e727-734.

27. Wocial L, Ackerman V, Leland B, Benneyworth B, Patel V, Tong Y, et al. Pediatric Ethics and Communication Excellence (PEACE) Rounds: Decreasing Moral Distress and Patient Length of Stay in the PICU. HEC forum : an interdisciplinary journal on hospitals' ethical and legal issues 2017;29(1):75-91. 


\section{Quick Look:}

\section{Current Knowledge:}

More children than ever are surviving critical illness with prolonged ventilation via tracheostomy tubes. However, these patients have a high complication rate, and the financial costs of their care is a large burden to the health care system.

\section{What this Paper Contributes to Our Knowledge:}

This study characterizes the patients requiring prolonged mechanical ventilation, quantifies the financial impact of their care, and describes their outcomes. It also compares these values for patients with primarily neurologic conditions vs. those with primarily respiratory conditions. 